\title{
Brachial Plexus Schwannoma: Case Report
}

\author{
S Touihmi ${ }^{1,2 *}$, Hicham Mimouni ${ }^{1,2}$ and Ilham Rkain ${ }^{1,2}$ \\ ${ }^{1}$ Faculty of Medicine and pharmacy of tangier, Abdelmalek Saadi University, Tétouan, Morocco \\ ${ }^{2}$ Department of otorhinolaryngology, Head and Neck surgery, university hospital, Tanger, Morocco
}

*Corresponding author: S Touihmi, Faculty of Medicine and pharmacy of tangier, Abdelmalek Saadi University, Department of otorhinolaryngology, head and neck surgery, university hospital, Tanger, Morocco.

\begin{abstract}
Introduction: Brachial plexus schwannomas is a rare entity, only about $5 \%$ arise from the brachial plexus. The diagnosis can be a challenge to surgeons. we provide a case report of a brachial plexus schwannoma and we highlight the clinical aspects of these tumors, the role of imaging in the diagnosis, the surgical treatment, and its outcomes..

Case Report: A 62-year-old male patient, presented with a slowly growing neck mass that had been noted for more than 20 years, associated with slowly evolving weakness of the left upper limb. clinically a 4 x $5 \mathrm{~cm}$ mass located in the left supraclavicular region was found. (CT) showed a well- encapsulated, low-density mass near the cervical spinal root. The mass was excised through a supraclavicular incision. The histopathology of the tumor was consistent with Schwannoma. A neurological deficit of the left arm was found immediately after the operation. The patient received sessions of rehabilitation therapy only. Six months later, the motor neurological functions had partially recovered.
\end{abstract}

Discussion: Schwannomas are indolent tumors but may present with symptoms secondary to nerve compression. Clinically, the most common presenting symptoms are palpable mass, pain paresthesia/numbness, and weakness. Contrast-enhanced CT and magnetic resonance images (MRI) are helpful in the pre-operative diagnosis. MR imaging is the study of choice to delineate the margins of the tumor from surrounding tissues with the greatest contrast. The choice of surgical approach is important and can be selected according to the tumor size, tumor site, and relationship with adjacent organs. A variety of surgical approaches are used. Recently, intracapsular enucleation has been introduced to preserve the neurological functions, it is possible to open the capsule and shell out the tumor, thereby leaving the capsular nerve undisturbed and possibly avoiding functional deficits [1]. Despite the extrafascicular characterization of the tumor, it is almost always possible to find a small fascicle entering and exiting the proximal and distal poles of the tumors, such as in our reported case. This fascicle does not transmit nerve action potential and can be sectioned so that the tumor can be.

Keywords: Surgery; Brachial plexus schwannoma; Case report

\section{Introduction}

Schwannomas, also known as neurinomas, are benign, solitary nerve sheath neoplasm. When they occur in the head and neck, in $25-45 \%$ of cases, they usually develop in cranial nerves and the sympathetic chain with relative sparing of the brachial plexus. Brachial plexus tumors are a rare entity, only about 5\% arise from the brachial plexus [2]. Due to their rarity and complex anatomical location they can pose a formidable challenge to surgeons. We provide a summary of the brachial plexus schwannoma, and we highlight the clinical aspects of these tumors, the role of imaging in the diagnosis, the surgical treatment and its outcomes.

\section{Case Report}

A 62-year-old male patient, with no personal or family history of malignancy and no particular medical features, came to our department for further work-up of a left-sided neck mass. The slowly growing neck mass had been noted for more than 20 years. The swelling was painless but gradually increasing in size, associated with the slowly evolving weakness of the left upper limb. Otherwise, he negated any tingling, numbness in his arm. Physical examination revealed a $4 \times 5 \mathrm{~cm}$ mass located in the left supraclavicular region. The mass was firm, semi-fixed and non- 
tender, and non-pulsatile. But the patient complained of a tingling sensation and electric discharge over the left upper arm when the mass was compressed. Other ears, throat, and nose examinations were normal. Neurological examination showed the slight weakness of the left arm, No café-au lait spot was observed.

(CT) showed a well-encapsulated, low-density mass near the cervical spinal root (Figure 1). Under general anesethesia, the mass was excised through a supraclavicular incision. A well-encapsulated yellow-brown mass over which several nerve fascicles spread was found during the operation (Figure 2). The nerves were carefully dissected from the mass except for one nerve running through the mass was sacrificed to completely remove the tumor. Tracing these nerves, it was found that the tumor originated mainly from the lower trunk of brachial plexus (Figure 2). The histopathology of the tumor was consistent with Schwannoma. A neurological deficit of the left arm was found immediately after the operation. The patient received sessions of rehabilitation therapy only. Six months later, the motor neurological functions had partially recovered, no recurrence of the tumor was noted. (a)

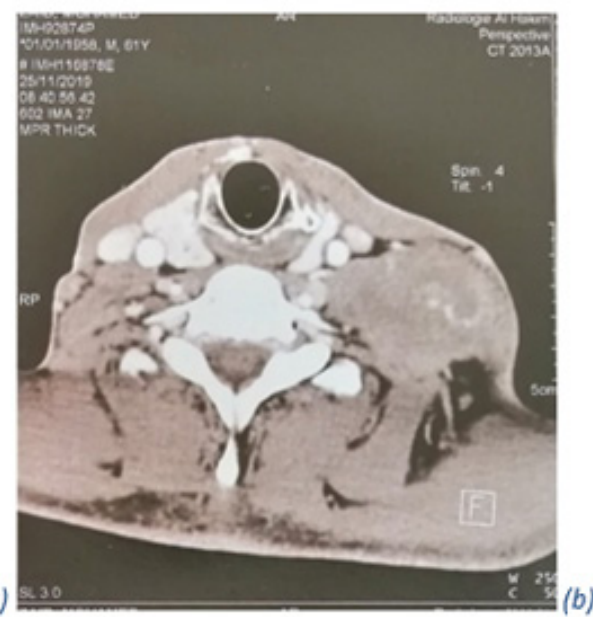

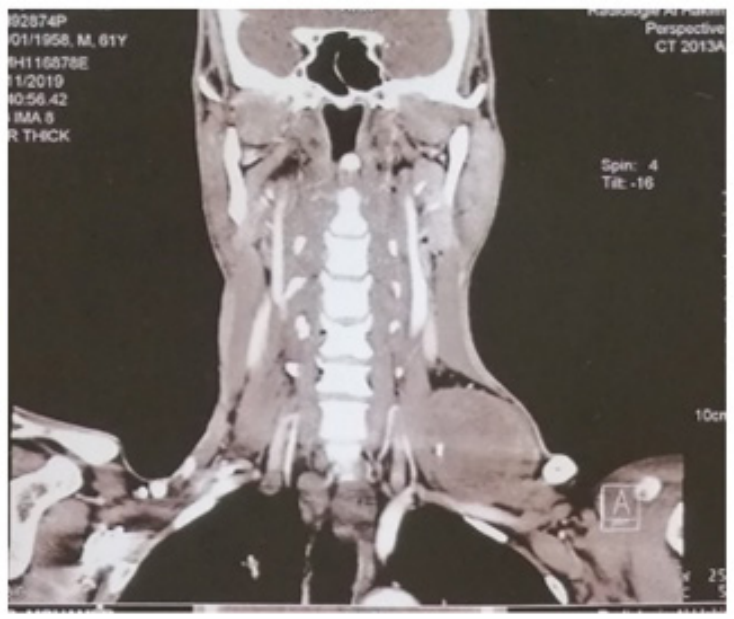

Figure 1: Axial (A) and coronal (B) computed tomography (CT) images showing well-circumscribed, oval, hypoattenuating left supraclavicular mass (arrows) with poor enhancement.
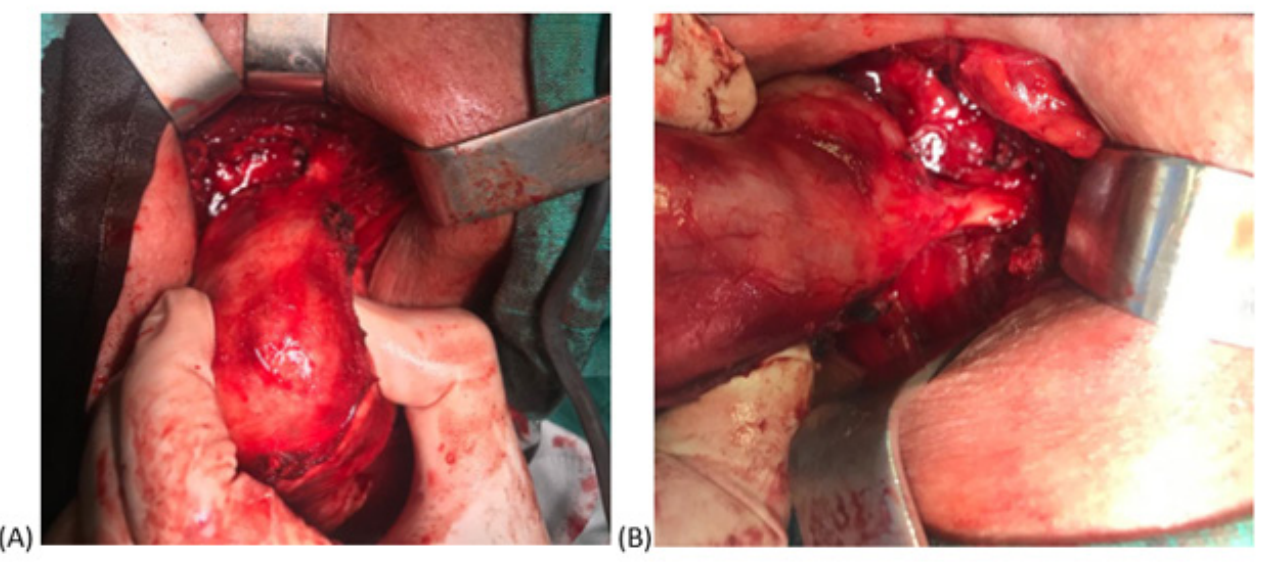

Figure 2: 2 Per-operative photograph of the tumour: Intraoperative details of schwannoma brachial plexus. (A) and (B) Well encapsulated mass splitting the fascicles of brachial plexus, (B) schwannoma after careful separation from fascicles expect for one nerve running through the mass.

\section{Discussion}

Schwannomas are the most frequent and largest category of benign nerve tumors. They are variously referred to as neuromas, neurinomas, and neurilemmomas in the literature. Brachial plexus tumors are rare, comprising only $5 \%$ of all tumors of the upper limb [3]. It commonly appears after the fourth decade of life but can occur at any age. there is no sex predisposition. Schwannomas of the brachial plexus, unassociated with von Recklinghausen's disease, are rarely encountered [4]. Though benign, they can undergo malignant transformation [5]. Schwannomas usually arise spontaneously, although, they are a principal feature of two hereditary tumor diseases, neurofibromatosis type 2 and schwannomatosis [6]. Schwannomas are indolent tumors but may present with symptoms secondary to nerve compression. Clinically. 
The most common presenting symptom are palpable mass, pain paresthesia/numbness, and weakness (1.11.20). In agreement with the symptomatology in our case, in general, pain is correlated with malignancy [7].

Most often, early in the course, the minimal neurological deficit will be present or there will be none at all. Manipulation of the mass can produce paresthesias or "shocks" in the distribution of the affected nerve: tinel sign, which carries a high predictive value for schwannoma, and this can be an important aid to diagnosis. The Side to-side mobility greater than longitudinal mobility of the mass from the nerve is more common in cases of benign lesions. Pre-operative diagnosis of schwannomas is difficult and several differential diagnoses for tumor of the neck may be considered, including paraganglioma, branchial cleft cyst malignant lymphoma and metastatic cervical lymphadenopathy, angioma. Contrastenhanced CT and magnetic resonance images (MRI) are helpful in the pre-operative diagnosis. MR imaging is the study of choice to delineate the margins of the tumor from surrounding tissues with the greatest contrast [8]. However, MR imaging is currently unable to differentiate between schwannoma and neurofibroma [9], generally, schwannomas are hypointense on T1 and hyperintense onT2-weighted images, depending on its cellularity. In our case, and due to the lack of recourses, only a computed tomography scan was done to assess the tumor. On computed tomography scan, schwannoma appears as a well and fusiform mass with a relatively homogenous contrast enhancement with internal cystic changes that become more prominent as the tumor enlarges. This cystic change is associated with mucinous degeneration, hemorrhage, necrosis, and microcystic formation.

The usefulness of fine-needle aspiration and cytology is still controversial; the majority of authors do not recommend open or needle biopsy for these masses. Besides making the removal of the tumor mass difficult by the incisional biopsy, no conclusive diagnosis can be obtained [10-12]. Histologically, schwannomas exhibit distinctive cylindrical structures and tendency towards palisading of the nuclei (Verocay bodies) which called Antoni A tissue, and a nondistinctive, loose stroma of fibers cells called Antoni B tissue. The therapeutic management of the schwannoma is a complete surgical excision, these tumors are relatively radioresistant $[4,10]$. Total extirpation of the tumor, with preservation of intact fascicles, is always the goal for surgical treatment. Incomplete treatment, such as an open biopsy or exploration without tumor removal should not be performed. Open incisional biopsy often results in peritumoral scar formation, which can obscure the surgical plane, and thus, render subsequent tumor resection more difficult [13]. The choice of surgical approach is important and can be selected according to the tumor size, tumor site, and relationship with adjacent organs. A variety of surgical approaches are used, including anterior supraclavicular or infraclavicular, combined/transclavicular, posterior subscapular, transaxillary approach $[14,15]$.
Recently, intracapsular enucleation has been introduced to preserve the neurological functions, it is possible to open the capsule and shell out the tumor, thereby leaving the capsular nerve undisturbed and possibly avoiding functional deficits [11]. Despite the extrafascicular characterization of the tumor, it is almost always possible to find a small fascicle entering and exiting the proximal and distal poles of the tumors, such as in our reported case. This fascicle does not transmit nerve action potential and can be sectioned so that the tumor can be removed. Our operative approach involves extracapsular excision, but some authors prefer intracapsular enucleation. Serious complications due to brachial plexus operations are infrequent. The primary risk is of neurological dysfunction caused by interruption of critical motor branches to arm and hand [16], Transitory nerve paresis may occur even when benign tumors are carefully dissected [6]. Generally, the larger tumors were the more is associated with a greater risk of neurologic deficit after surgery [13, 17-21].

\section{Conclusion}

The key element in management is a correct pre-operative diagnosis; dealing with cases of the neck mass, the possibility of a schwannoma should be considered, especially when there are some existing neurological symptoms. Imaging studies such as CT or MRI should be done for pre-operative assessment. During the operation, care should be taken to preserve nerve function Neurological deficit may sometimes be inevitable when removing the mass, thus the doctor should discuss the possibility of a neurological deficit with the patient before the operation.

\section{Acknowledgement}

None.

\section{Conflict of Interest}

No Conflict of Interest.

\section{References}

1. Ines Nacef, Skander Kedous, Zied Attia, Slim Touati, Said Gritli (2004) Cervical sympathetic chain schwannoma: A case report. Egyptian Journal of Ear, Nose, Throat and Allied.

2. Akhtar, Ameet Kumar, Saeed (2011) Schwannoma of Brachial Plexus. Indian J Surg.

3. Devin K Binder, Justin S. Smith (2004) Primary brachial plexus tumors: imaging, surgical. Neurosurg Focus 16 (5): E11.

4. Dhasmana, Sunil Saini and Janardan P (2006) Apical Mass Presentation of Brachial Plexus Schwannoma. The journal of bone and Joint surgery.

5. Luka Vučemilo, Zoran Lajtman (2018) Brachial Plexus Schwannoma Case Report and Literature Review. Acta Clin Croat 57(2): 366-371.

6. Stephen T Wysong, Michael J Boros (2012) Syndromes after resection of a cervical schwannoma. Ent journal.

7. Jason H Huang, Kareem Zaghloul, Eric L Zager (2004) Surgical Management of Brachial. Surg Neurol.

8. Masaru Inoue, Teruaki Kawano, Hidekatsu Matsumura (2000) Solitary Benign Schwannoma of the Brachial Plexus. Surg Neurol 20(2): 103-108. 
9. Hyuk Jin Lee, Jeong Hwan Kim (2014) Is Surgery for Brachial Plexus Schwannomas Safe and Effective? Clin Orthop Relat Res 472: 1893-1898.

10. Young Woo Kim, Sung Ki Ahn, Joon Ho Song (2006) A Case of Brachial Plexus Schwannoma. J Korean Neurosurg Soc 39: 396-399.

11. Knight DMA (2006) Benign solitary schwannoma: report of 244 Cases. The journal of bone and Joint surgery.

12. Luka Vučemilo, Zoran Lajtman (2018) Brachial Plexus Schwannoma Case Report and Literature Review. Acta Clin Croat 57(2): 366-371.

13. Lusk Micheal D (2013) Tumors of the brachial plexus. Neurosurgery 21(4): 439-453.

14. Mark K Wax (2004) Cervical Sympathetic Chain Schwannoma. The American Laryngological.

15. Richardson, Robert R (2001) Neurogenic tumor of brachial plexus: Report of two cases. Neurosurgery 4(1): 66-70.
16. Munna Lal Patel, Rekha Sachan, Ganesh Seth, Radheshyam, Patel ML, et al. (2013) Schwannoma of the brachial plexus: a rare cause of monoparesis. BMJ Case Rep.

17. Uzun Lokman (2005) CERVICAL sypmathic chain schwannoma mimicking a carotid body tumor: A case report. Tumori 91(1): 84-86.

18. Kyeong Hwa Ryu (2000) Brachial plexus schwannoma mimicking cervical lymphadenopathy/A case report with emphasis on imaging features. Clinical Case Report.

19. Hui-chi Ku, Chi-wei Yeh (2010) Cervical schwannoma: a case report and eight years review. J Laryngol Otol 114(6): 414-417.

20. Jaafar Rohazam (2012) Cervical scwannoma: Report of four cases. Med J Malaysia 67(3): 345-348.

21. Naoa EEM (2012) Cervical sympathetic chain schwannoma. European annalas of Otoryhinolaryngology head and neck disease. 129(1): 51-53. 